\title{
INJECTIVE HULLS AS COMPLETIONS
}

\author{
by PAUL D. BACSICH $\dagger$
}

(Received 23 January, 1970; revised 28 November, 1970)

A preliminary attempt is made to place the theory of completions of boolean algebras and of partially ordered sets in a wider context. The theory and construction of injective hulls in abelian categories is generalised and it is demonstrated that any variety with enough injectives admits injective hulls. Then the methods developed are applied to a non-algebraic bicategory, that of ordered sets.

1. Bicategories. We assume that the reader is familiar with the elementary aspects of the theory of bicategories as expounded in Isbell [12] and Semadeni [16].

Let $K$ be a bicategory. We recall that an object $A$ of $K$ is said to be injective if whenever $e: B \rightarrow C$ is an injection and $f \in \operatorname{Hom}(B, A)$, then there is $h \in \operatorname{Hom}(C, A)$ such that $h e=f$. An injection $e: A \rightarrow A^{\prime}$ is called proper if it is not an equivalence, and dense if, whenever $f \in \operatorname{Hom}\left(A^{\prime}, B\right)$ is such that $f e: A \rightarrow B$ is an injection, then $f$ is an injection. $A^{\prime}$ is called an extension of $A$ by $e$ if $e: A \rightarrow A^{\prime}$ is an injection.

In this section we state a number of simple lemmas which are necessary for the proof of the main theorem in $\S 2$. Proofs for the first two are supplied so as to establish the techniques to use in the nonabelian case, and using these the other lemmas should be obvious. (A treatment of the abelian case can be found in [14], Section III.2.)

LEMma 1.1. The composition of dense injections is a dense injection.

Proof. Let $e: A \rightarrow B, f: B \rightarrow C$ be dense injections. Then $f e: A \rightarrow C$ is an injection. Let $g \in \operatorname{Hom}(C, D)$ be such that $g f e$ is an injection. Then $g f$ is an injection as $e$ is dense, and so $g$ is an injection as $f$ is dense.

Lemma 1.2. Let e: $A \rightarrow B, e^{\prime}: A \rightarrow C$ be dense injections, $f \in \operatorname{Hom}(B, C)$ be such that $f e=e^{\prime}$. Then $f$ is a dense injection.

Proof. $f$ is an injection, as $e$ is dense. Now let $g \in \operatorname{Hom}(C, D)$ be such that $g f$ is an injection. Then $g f e=g e^{\prime}$ is an injection, and so $g$ is an injection as $e^{\prime}$ is dense.

DEFINITION 1.3.

(1) A pair $\left(e, A^{\prime}\right)$ is an injective extension of $A$ if $A^{\prime}$ is injective and $e: A \rightarrow A^{\prime}$ is an injection.

(2) An extension ( $\left.e, A^{\prime}\right)$ of $A$ is called dense if $e$ is dense.

(3) $\left(e, A^{\prime}\right)$ is an injective hull of $A$ if $\left(e, A^{\prime}\right)$ is a dense injective extension of $A$.

(4) A dense extension $\left(e, A^{\prime}\right)$ of $A$ is maximal if $A^{\prime}$ has no proper dense extension.

Lemma 1.4. Let $u: A \rightarrow B$ be a dense injection, $(e, D)$ an injective extension of $A$. Then there is an injection $e^{\prime}: B \rightarrow D$ such that $e^{\prime} u=e$.

By noting that a retraction is a surjection, we can establish

$\dagger$ During the period of research for this paper, the author was supported by a Science Research Council NATO Studentship at the University of Bristol. 
LEMMA 1.5. If $A$ is injective, then $A$ has no proper dense extensions.

LEMMA 1.6. Any two injective hulls of $A$ are isomorphic.

We say that $K$ has enough injectives if every object of $K$ has an injective extension. An injection $e: A \rightarrow D$ of $K$ is said to be reducible if there is a morphism $f: D \rightarrow B$ such that $f e: A \rightarrow B$ is a dense injection.

THEOREM 1.7. Suppose that $K$ has enough injectives and that every injection of $K$ is reducible. Let $A$ be an object of $K$. If $A$ has no proper dense extensions, then $A$ is injective.

Proof. Let $e: A \rightarrow D$ be an injective extension of $A$. Since $e$ is reducible, let $f: D \rightarrow B$ be a morphism such that $f e: A \rightarrow B$ is a dense injection. Hence $f e$ is an equivalence. Thus $A$ is a retract of $D$ by $(f e)^{-1} f$, and so $A$ is injective.

THEOREM 1.8. Suppose that $K$ has enough injectives. Then the following are equivalent:

(1) Every injection of $K$ is reducible and every object of $K$ has a maximal dense extension.

(2) Every object of $K$ has an injective hull.

2. Varieties. We recall that a variety is an equational class of algebras. It is known that any variety forms a bicategory in a natural way, with morphisms interpreted as homomorphisms, injections as embeddings (i.e. 1-1 homomorphisms), and surjections as surjective homomorphisms. We say that $A$ is a dense subalgebra of $B$ if $A$ is a subalgebra of $B$ and the inclusion map $A \rightarrow B$ is dense.

The following well-known property of varieties will be used implicitly several times: If $e: A \rightarrow B$ is an injection, then there is an algebra $A^{\prime}$ and an isomorphism $f: A^{\prime} \rightarrow B$ such that $A$ is a subalgebra of $A^{\prime}$ and $e=f \mid A$.

We first find a more algebraic characterisation of dense subalgebras.

Lemma 2.1. Let $K$ be a variety. Suppose that $A$ is a subalgebra of $B$. Then $A$ is a dense subalgebra of $B$ if and only if, for any congruence $J$ on $B$ with $J \cap A^{2}=\Delta_{A}$, we have $J=\Delta_{B}$ (where $\Delta_{A}$ is the graph of equality on $A$ ).

Proof. Let $e: A \rightarrow B$ be the inclusion map. Let $f \in \operatorname{Hom}(B, D), J$ be the kernel of $f$. Then $J \cap A^{2}$ is the kernel of $f e$. The lemma is now obtained by using the fact that a morphism $f^{\prime}: C \rightarrow C^{\prime}$ is an injection if and only if the kernel of $f^{\prime}$ is $\Delta_{C}$.

COROLlary 2.2. Suppose that $K$ is one of the following varieties: abelian groups, boolean algebras, commutative rings. Let $A$ be a subalgebra of $B$. Then $A$ is a dense subalgebra of $B$ if and only if, whenever $I$ is an ideal of $B$ such that $I \cap A=0$, then $I=0$.

As a further refinement we obtain the following result of [3].

COROLlaRY 2.3. Let $K$ be the variety of boolean algebras, $A$ be a subalgebra of $B$. Then $A$ is a dense subalgebra of $B$ if and only if $A$ is dense in $B$ in the usual boolean-algebraic sense that whenever $b \in B$ is nonzero, there is $a \in A$ such that $0<a \leqq b$.

The next result is Proposition VII.2.5 of Cohn [7], but we shall give a proof here as we wish to generalise the method later. 
THEOREM 2.4. Let $K$ be any variety. Then each injection of $K$ is reducible.

Proof. Let $A$ be a subalgebra of $D$. Consider the set $C$ of congruences $J$ on $D$ such that $J \cap A^{2}=\Delta_{A}$, ordered by inclusion. Then the union of any chain in $C$ belongs to $C$. Hence $C$ has a maximal member $J$, by Zorn's Lemma. Let $D / J$ be the quotient algebra of $D$ by $J$, $f: D \rightarrow D / J$ the canonical surjection. Let $e: A \rightarrow D$ be the inclusion map. Now $f e$ has kernel $J \cap A^{2}=\Delta_{A}$, so that $f e$ is an injection. Let $J^{\prime}$ be a nontrivial congruence on $D / J$, and let $J_{1}$ be the congruence on $D$ given by $d J_{1} d^{\prime}$ if and only if $f(d) J^{\prime} f\left(d^{\prime}\right)$. Then $J$ is a proper subset of $J_{1}$, and so there are distinct points $a, b \in A$ with $a J_{1} b$. For these points, $f(a) J^{\prime} f(b)$ and $f(a) \neq f(b)$. Hence $f e$ is a dense injection.

The next few results will establish that any variety with enough injectives admits injective hulls.

Lemma 2.5. Let $A$ be a subalgebra of an algebra $D$. Let $G$ be the collection of all subalgebras $C$ of $D$ such that $A$ is a dense subalgebra of $C$, ordered by inclusion. Then $G$ has a maximal member.

Proof. $G$ is nonvoid as $A \in G$. Let $\left(C_{i}: i \in T\right)$ be a chain in $G$. Then $C=\bigcup_{i \in T} C_{i}$ is a subalgebra of $D$, and also $C^{2}=\bigcup_{i \in T} C_{i}{ }^{2}$, since we have a chain. Now let $J$ be a congruence on $C$ such that $J \cap A^{2}=\Delta_{A}$. Then $J=\bigcup_{i \in T}\left(J \cap C_{i}{ }^{2}\right)$, and $J \cap C_{i}{ }^{2}$ is a congruence on $C_{l}$ with $\left(J \cap C_{i}{ }^{2}\right) \cap A^{2}=\Delta_{A}$, so that $J \cap C_{i}{ }^{2}$ is the diagonal on $C_{i}$. Hence $J=\Delta_{C}$. This shows that $A$ is a dense subalgebra of $C$. Thus the union of a chain in $G$ belongs to $G$, and so, by Zorn's Lemma, $G$ has a maximal member.

Lemma 2.6. Let $K$ be a variety with enough injectives, $A$ an algebra of $K$. Then $A$ has a maximal dense extension.

Proof. Let $D$ be an injective algebra of $K$ such that $A$ is a subalgebra of $D$. Define $G$ as in Lemma 2.5 and let $B$ be a maximal member of $G$. We show that $B$ has no proper dense extension. Let $\left(e, B^{\prime}\right)$ be a dense extension of $B$. Then there is an injection $f: B^{\prime} \rightarrow D$ with $f e(b)=b$ for $b \in B$, by Lemma 1.4. Let $B^{\prime \prime}$ be the image of $B^{\prime}$ under $f$. Then $B$ is a subalgebra of $B^{\prime \prime}$ and $B^{\prime \prime}$ is a subalgebra of $D$. Now $B$ is a dense subalgebra of $B^{\prime \prime}$ (as $B^{\prime \prime}$ is isomorphic to $B^{\prime}$ ) and so $A$ is a dense subalgebra of $B^{\prime \prime}$, by Lemma 1.1. Thus $B=B^{\prime \prime}$, and so $f$ is an isomorphism of $B^{\prime}$ onto $B$. Hence $e: B \rightarrow B^{\prime}$ is an isomorphism, and so $B$ is a maximal dense extension of $A$.

THEOREM 2.7. Let $K$ be a variety with enough injectives. Then every algebra of $K$ has an injective hull.

Proof. Use Lemma 2.6 and Theorem 2.4, and then Theorem 1.8.

We observe that, if $K$ has an injective separator $D$ (in the sense of 5.1 of [16]), then $K$ has enough injectives. For let $A$ be an algebra of $K, I=\operatorname{Hom}(A, D)$, and $e: A \rightarrow D^{I}$ be given by $e(a)(m)=m(a)$ for $m \in I, a \in A$. Then $e$ is an injection, while $D^{I}$ is injective. Hence the following varieties admit injective hulls (the algebras in parentheses are injective separators):

(1) Abelian groups (the circle group)-by $\S 9.4$ of [16], 
(2) Boolean algebras (the 2-element algebra), by Column 8 of the Table in [16],

(3) Distributive lattices with 0 and 1 (the 2-element lattice)-by Lemma 3.1 of [2],

(4) Semilattices with 0 and 1 (the 2-element semilattice), by the remark after Corollary 4.7,

(5) $p$-rings with 1 (the finite field with $p$ elements), for prime $p$-by $\S 5$ of [13].

A general treatment of injective hulls in abelian categories will be found in Freyd [9] (note that only (1) above is abelian).

3. Boolean algebras. Let us consider the variety of boolean algebras. By replacing congruences by ideals and making the obvious adjustments in Theorem 2.4 and Lemmas 2.5 and 2.6, we obtain a very simple proof of the result: Every boolean algebra has an injective hull. It now follows that every complete boolean algebra is injective, by using the following two lemmas, the first due to Halmos [11] and the second to Sikorski [18, Theorem 23.1].

Lemma 3.1. Every injective boolean algebra is complete.

Proof. Every such is a retract of some $2^{I}$ and so is complete.

LEMmA 3.2. Let $B$ be a complete boolean algebra, $A$ a dense subalgebra of $B$. Then $A$ is $a$ regular subalgebra of $B$, and, for $b \in B, b=\sup _{B}\{a \in A: a \leqq b\}$. obvious.

Thus, if $A$ is itself complete, $A=B$. By applying Theorem 2.7, the next theorem is

THEOREM 3.3. Every complete boolean algebra is injective.

This result was first proved by Sikorski in [17], using step-by-step extension and Zorn's Lemma. Gleason [10] has given a proof using the Stone duality, and Scott [15] has proved it using Boolean-valued models of set theory. The above technique of deriving Theorem 3.3 is due, in dual form, to Gleason (see Lemma 2.3 and Theorem 2.5 of [10]). It first appeared explicitly in [8].

Lemma 3.2 (together with Corollary 2.3) also shows that there is a general-algebraic proof of the existence of the completion of a boolean algebra $A$. For any injective hull of $A$ clearly has properties (i), (iii), and (iv) of Theorem 35.2 in [18].

4. Partially ordered sets. Let $K$ be the category of (partially) ordered sets and orderpreserving maps. This can be made into a bicategory by defining $e: A \rightarrow B$ to be an injection precisely if $e$ is an order-isomorphism of $A$ onto a subset of $B$. By adapting the abstract construction of $\S 2$, we shall show that $K$ admits injective hulls, and then these will be characterised as Dedekind-MacNeille completions.

Our first task is to show that the algebraic notions of congruence and kernel have suitable analogues in $K$. We recall that a preorder is a reflexive transitive relation.

Lemma 4.1. Let $f:(A, \leqq) \rightarrow(B, \leqq ')$ be a morphism of $K$. Define a relation $\leqq$ on $A$ as follows: $x \leqq_{f} y$ if and only if $f(x) \leqq^{\prime} f(y)$. Then $\leqq_{f}$ is a preorder on $A$ extending $\leqq$, and $f$ is an injection if and only if $\leqq_{f}=\leqq$ (as subsets of $\left.A^{2}\right)$. We call $\leqq$ the kernel of $f$.

Proof. If $x \leqq y$, then $f(x) \leqq f^{\prime}(y)$ and so $x \leqq f_{f} y$. Hence $\leqq \subseteq \leqq$. Clearly $\leqq_{f}$ is a 
preorder. The proof is completed by recalling that $f$ is an injection if and only if, for all $x, y \in A, x \leqq y$ if and only if $f(x) \leqq \prime f(y)$.

LEMMA 4.2. Let $\leqq$ be a preorder on $A$. Define a relation $E$ on $A$ by $x E y$ if and only if $x \leqq y$ and $y \leqq x$. Then $E$ is an equivalence and $A / E$ admits a natural partial order $\varliminf^{0}$ such that $x \leqq y$ if and only if $\bar{x} \leqq{ }^{0} \bar{y}$, where $\bar{x}$ is the E-equivalence class of $x$, for $x \in A$. Further, if $\leqq 1$ is a partial order on $A$ with $\varliminf_{1} \subseteq \leqq$, then the canonical map $f:\left(A, \leqq_{1}\right) \rightarrow\left(A / E\right.$, $\left.{ }^{0}\right)$ is orderpreserving and $\leqq \mathrm{f}=\leqq$.

Lemma 4.3. Let $(B, \leqq)$ be an ordered set, $A$ a subset of $B, \leqq_{A}$ the restriction of $\leqq$ to $A$. Then the inclusion map $e:\left(A, \leqq_{A}\right) \rightarrow(B, \leqq)$ is dense if and only if, for every preorder $R$ on $B$ extending $\leqq$, if $R \cap A^{2}=\leqq$, then $R=\leqq$.

Proof. Let $f:(B, \leqq) \rightarrow\left(C, \varliminf^{\prime}\right)$ be order-preserving. Now it is sufficient to remark that $f e$ is an injection if and only if $\leqq_{f e}=\leqq_{A}$, and that $\leqq_{f e}=\leqq_{f} \cap A^{2}$.

THEOREM 4.4. Every injection of $K$ is reducible.

Proof. Let $e$ be an injection of $K$. We may assume that $e$ is an inclusion map $A \rightarrow B$. Let $R$ be a preorder on $B$ maximal such that $\leqq \subseteq R$ and $R \cap A^{2}=\leqq_{A}$ (by using a proof similar to that of Theorem 2.4). Let $f: B \rightarrow C$ be a morphism with kernel $R$. Then $f e$ is a dense injection (proof as in Theorem 2.4).

The next two lemmas will demonstrate that $K$ has enough injectives. Lemma 4.5 is a result of [3].

LeMma 4.5. Every complete lattice is injective in $K$.

Proof. Let $L$ be a complete lattice, $B$ an ordered set, $A$ a subset of $B, f \in \operatorname{Hom}(A, L)$. Define $g: B \rightarrow L$ by $g(x)=\sup \{f(a): a \in A$ and $a \leqq x\}$. Then $g$ extends $f$ and is orderpreserving.

Lemma 4.6. Every ordered set $P$ is order-embeddable in a complete atomic boolean algebra.

Proof. This is classical: we can embed $P$ in the power set of $P$.

COROLlaRY 4.7. Every injective in $K$ is a complete lattice.

Proof. Use Lemma 4.6 and an argument similar to that for Lemma 3.1.

Essentially the same methods will show that in the variety of (meet) semilattices with 0 and 1 , the injectives are precisely the complete Brouwerian lattices (by noting that the embedding of Lemma 4.6 preserves meets and that a meet-retract of a boolean algebra is pseudo-complemented-this has been observed independently in [5]). Thus the 2-element semilattice is an injective separator.

THEOREM 4.8. $K$ admits injective hulls.

Proof. Let $A$ be an object of $K$. Let $A$ be a subset of $D$, where $D$ is injective (by Lemmas 4.6 and 4.5). By carrying out the proof of Lemma 2.5 using preorders in place of congruences, and using an argument similar to that in Lemma 2.6, we can show that $A$ has a maximal dense extension. But this is injective by Theorem 1.8. 
We now show that injective hulls have a concrete realisation as (Dedekind-MacNeille) completions. The standard construction of the completion can be found in Birkhoff [4].

THEOREM 4.9. Let $A$ be an ordered set. Then any injective hull of $A$ is a completion.

Proof. Let $L \supseteq A$ be an injective hull of $A$. Then $L$ is a complete lattice by Corollary 4.7. Also, there is a preorder $R$ on $L$ extending $\leqq_{L}$, given by $x R y$ if and only if $I_{x} \subseteq I_{y}$, where $I_{x}=\{a \in A: a \leqq x\}$. Now $R=\leqq_{L}$ as $R \cap A^{2}=\leqq_{A}$. Thus, for $x \in L, x=\sup I_{x}$, and the dual of this also holds.

Note that as we wish to construct the completion, we cannot use Proposition 4.2 of [3], which assumes its existence.

By dualising the definitions of $\S 1$, we arrive at the notions of dense surjection and projective cover. We now show that $K$ admits projective covers. An object $A$ of $K$ is said to be free if $A$ is totally unordered. The reader can easily check the following facts:

(1) Every free object is projective.

(2) Every object is a quotient of a free object.

(3) Every projective object is free.

(4) A natural projective cover of $(A, \leqq)$ is $\left(u,\left(A, \leqq_{1}\right)\right)$, where $\leqq_{1}$ is equality on $A$ and $u$ is the identity map.

5. Concluding remarks. The methods developed here can also be applied to give a short abstract proof of Cohen's result (see [6]) that the bicategory $K$ of normed linear spaces and linear contractions admits injective hulls. For $K$ has enough injectives (by Column 11 of the Table in [16]), and by replacing congruences by seminorms the obvious analogues of Theorem 2.4 and Lemmas 2.5 and 2.6 can easily be obtained.

It has been brought to the notice of the author that there is a general construction of injective hulls in Daigneault [8]. However, this would not apply to partially ordered sets or normed linear spaces, and for varieties, it leads to a more complicated proof (as the conditions $\mathrm{C} 3$ and $\mathrm{C} 6$ of [8] are nontrivial to verify in general).

An abstract approach to injective hull constructions and further investigation of completions (e.g. in metric spaces) can be found in [1].

\section{REFERENCES}

1. P. Bacsich, Algebraic injectivity and weak choice axioms, Ph.D. Thesis, University of Bristol, 1970.

2. R. Balbes, Projective and injective distributive lattices, Pacific J. Math. 21 (1967), 405-420.

3. B. Banaschewski and G. Bruns, Categorical characterisation of the MacNeille completion, Arch. Math. Basel 18 (1967), 369-377.

4. G. Birkhoff, Lattice theory, Amer. Math. Soc. Colloquium Publications 25 (Providence, R.I., 1967).

5. G. Bruns and H. Lakser, Injective hulls of semilattices, Canad. Math. Bull. 13 (1970), 115-118.

6. H. Cohen, Injective envelopes of Banach spaces, Bull. Amer. Math. Soc. 70 (1964), 723-726.

7. P. Cohn, Universal algebra (New York, 1965).

8. A. Daigneault, Injective envelopes, Amer. Math. Monthly 76 (1969), 766-774. 
9. P. Freyd, Abelian categories (New York, 1964).

10. A. Gleason, Projective topological spaces, Illinois J. Math. 2 (1958), 482-489.

11. P. Halmos, Lectures on Boolean algebras (Princeton, 1963).

12. J. Isbell, Algebras of uniformly continuous functions, Ann. of Math. 68 (1958), 96-125.

13. N. McCoy and D. Montgomery, A representation of generalised Boolean rings, Duke Math. J. 3 (1937), 455-459.

14. B. Mitchell, Theory of categories (New York, 1965).

15. D. Scott, Lectures on Boolean-valued models for set theory, Summer Institute in Set Theory, Los Angeles, 1967 (to appear).

16. Z. Semadeni, Projectivity, injectivity and duality, Rozprawy Matematyczne 35 (1963).

17. R. Sikorski, A theorem on extension of homomorphisms, Ann. Soc. Pol. Math. 21 (1948), 332-335.

18. R. Sikorski, Boolean algebras, 2nd edn (Berlin, 1964).

Mathematical InSTItUTE

OXFORD UNIVERSITY 